\title{
Abscopal effect in the radio and immunotherapy
}

\author{
Alina M. Pevzner, Matvey M. Tsyganov, Marina K. Ibragimova, Nikolai V. Litvyakov \\ Research Institute of Oncology, Tomsk National Research Medical Center of the Russian Academy of Sciences, Tomsk, Russia
}

Received: January 21, 2021

Revised: May 27, 2021

Accepted: May 31, 2021

\section{Correspondence:}

Matvey M. Tsyganov

Research Institute of Oncology, Tomsk

NRMC, Tomsk, st. Cooperative 5,

634009, Russia.

Tel: +7-3822-282676

E-mail:TsyganovMM@yandex.ru

ORCID:

https://orcid.org/0000-0001-7419-4512
This review is devoted to a rare in clinical practice, but promising phenomenon of regression distant non-irradiated metastases in combination therapy of cancer patients. R. H. Mole in 1953 suggested introducing the term "abscopal effect" to denote the effect of ionizing radiation "at a distance from the irradiated volume but within the same organism." Currently, it is a hypothesis in the treatment of metastatic cancer, when there is a regression of untreated areas simultaneously with a decrease in the tumor. After the discovery of immune checkpoint cases were increase with patients treated with check-point blockade (especially lymphocyte associated protein 4, programmed cell death 1/programmed cell death 1 ligand 1) and which have an abscopal effect. This review systematizes works covering the time period from 1969 to 2019, which give cases of the abscopal effect at different localizations. However, abscopal effect is a poorly understood phenomenon. In this review, the authors tried to collect all information about the possible mechanisms of the abscopal effect, possible role in antitumor response and frequency abscopal effect at radio/immunotherapy or combined both.

Keywords: Abscopal effect, Radiotherapy, Immunotherapy, Cancer

\section{Introduction}

Radiation therapy (RT) is used to reduce the risk of relapse after surgery, as a means for reduction of tumor volume in palliative care, or as the main method of treatment for some tumors. As a rule, RT for cancer patients involves local irradiation of the tumor node. However, the researchers have been observed cases of "distant" effects of radiotherapy, in particular regression of hematogenous metastases, with local irradiation of the primary tumor node since the beginning of the 20th century.

The first complete regression of the tumor was noted in 1908 when the effect of radiation was studied on lymph nodes remote from the main focus. Unfortunately, in this study there was no exact description of the irradiation procedure, dose, and localization of the irradiated lymph nodes, which cannot exclude the influence of other factors on the reached effect [1]. In 1938, an "irregular reaction" (adrenal hypertrophy and atrophy of lymphoid organs) was observed in unirradiated organs in animal model in vivo, when a tumor was irradiated 20-40 Gy [2]. Fifteen years later, in 1953, Mole
[3] suggested introducing the term "abscopal effect" to denote the effect of ionizing radiation "at a distance from the irradiated volume but within the same organism," and then expanded to include distant effects on normal tissues [4]. Currently, it is a hypothesis in the treatment of metastatic cancer, when there is a regression of untreated areas simultaneously with a decrease in the tumor. This means that localized radiation initiated an antitumor response, which kills cancer cells remote from the main target. Despite the fact that the first recorded clinical cases of tumor regression after chemotherapy and RT date back to the beginning of the 20th century, the abscopal effect is still quite rare in clinical practice [4].

It is important to note that cases of tumor regression were also observed in cancer patients with various infectious diseases [5]. American surgeon Coley [6] was convinced that having a severe infection could cause tumor regression. His experiments showed that the inactivated pathogens of Streptococcus pyogenes in the vaccinated patient could not cause an infectious disease, but nevertheless all the signs of inflammation developed, and the body began to fight and the tumor disappeared. Interestingly, in experiments in 
vitro, bacterial strains did not affect tumor cells in any way. Therefore it was concluded that the infection did not directly impact on the tumor, but activated the patient's immunity, which led to the regression of the tumor node.

Scientists have argued over the role of the immune system in the antitumor response over a long period of time. More than 30 years ago, Stone et al. [7] showed that there is a lack of tumor response to RT with the deficiency of a normal pool of T cells. It is now well known that tumor progression is associated with the development of many immunosuppressive mechanisms that allow cancer cells to escape immune control. Consider that RT also has an immunosuppressive effect and is not able to induce an effective antitumor immune response, which leads to the destruction of the tumor. However, in some cases, immunosuppression and the development of an antitumor immune response as an abscopal effect. In this case, stimulation of the patient's immunity (through immunotherapy) and/or overcoming of tumor-induced immunosuppression during RT should provide an increase in the latter's effectiveness and an increase in the frequency of the abscopal effect.

\section{Mechanism of the Abscopal Effect}

It is believed that RT has an immunosuppressive effect, leads to the suppression of co-stimulatory surface markers CD80 and CD86 on native dendritic cells, thereby inhibiting the activation of T cells [8]. However, cell death caused by RT stimulates many immune responses and changes the tolerant tumor phenotype [9]. During radiation therapy, a special functional type of cell apoptosis can be activated-immunogenic cell death (ICD), which is accompanied by stimulation of antigen-specific adaptive immunity [10]. ICD leads to many antitumor immune responses: the release of tumor antigens by irradiated tumor cells, the cross-presentation of antigens originating from the tumor to T cells using antigen-presenting cells, and the migration of effector $T$ cells from lymph nodes to distant tumor [11]. In this regard, the number of studies on the effect of RT on various immune responses (activation of antitumor immune responses) and the abscopal effect has increased. In a recent review that covered the time period from 1969 to 2014, 46 cases of the demonstration of an abscopal effect in patients after RT were described [12]. Moreover, the frequency of occurrence of the RT-induced abscopal effect is very low [13]. However, the documented occurrence of this effect in RT provides an incentive for a more thorough further study.

At present, the following data on the mechanisms of the abscopal effect are available. When exposed to tumor cells in RT, the dying tumor cells have a huge number of changes and begin to actively express damage-associated molecular patterns (DAMP), calreticulin, adenosine triphosphate, high mobility group box 1 (HMGB1) protein, type I interferons, nucleic acids derived from cancer cells, annexin A1, etc. [14], releasing a large number of tumor antigens (Fig. 1). Antigen-presenting cells, such as dendritic cells and phagocytic cells, interact with the emerging tumor antigens and then migrate to the lymph nodes. The presentation of antigens occurs through the major histocompatibility complex (MHC), through the T cell receptor (TCR) [15]. However, antigenic complex interactions only through the TCR are not enough to activate T cells. Other co-stimulating signals are needed, such as CD80, CD86, and CD28 [16]. If activation by several T cell signals occurred (there was no inhibition on the part of the tumor), then many types of $T$ cells and, especially, CD8+ T cells, which play the main role in the

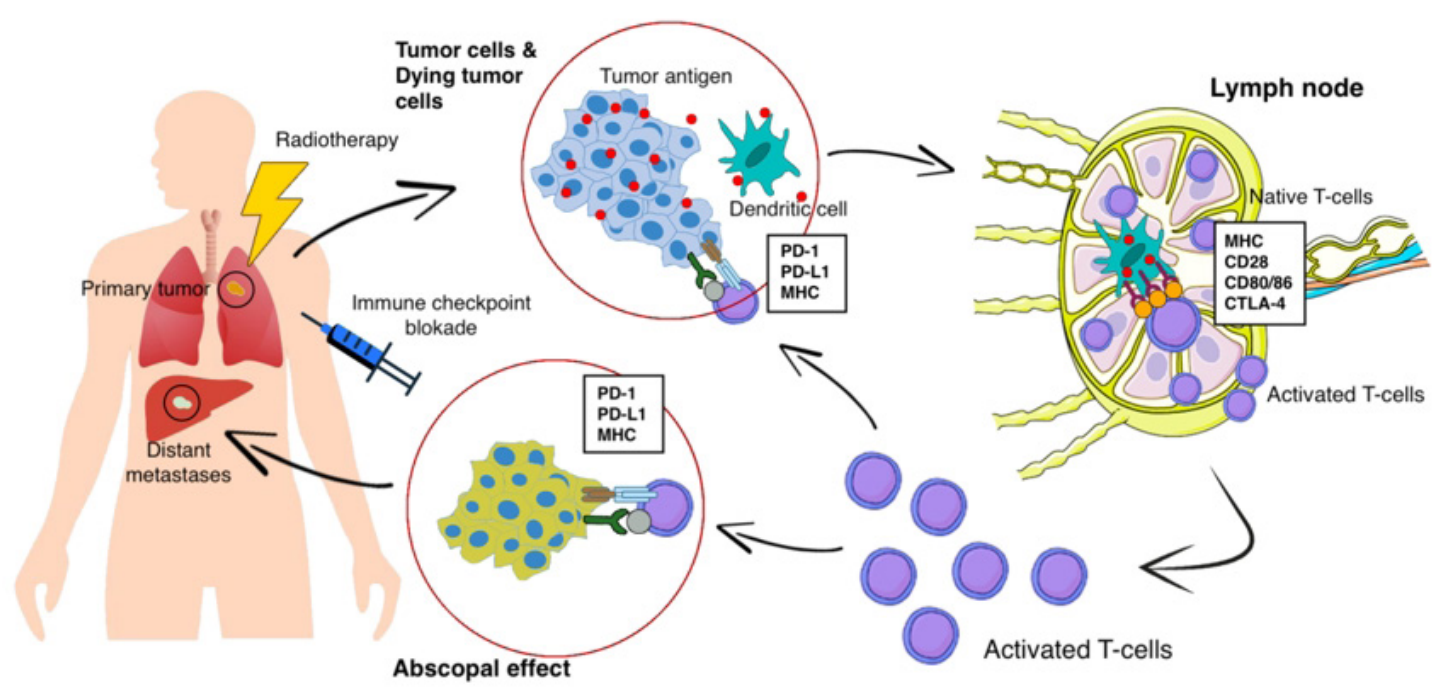

Fig. 1. The mechanism of radiotherapy-induced abscopal effect combined with immunotherapy. PD-1, programmed cell death 1; PD-L1, programmed cell death 1 ligand 1; MHC, the major histocompatibility complex; CTLA4, cytotoxic T-lymphocyte associated protein 4. 
antitumor immune response, are activated and begin to reproduce. As a result, activated effector T cells exit the lymph nodes, and interactions with TCR affect the primary tumor and unirradiated distant metastasis [16]. Presumably, such a mechanism can be used to explain the abscopal effect in distant metastases with local tumor irradiation. Preclinical studies also show a clear role for the immune system in the manifestation of the abscopal effect. Demaria et al. [17] found that T cells mediate distant tumor inhibition caused by RT. Work on a mouse model of breast cancer showed a decrease in pulmonary metastases and improved survival only in mice treated with RT in combination with cytotoxic T-lymphocyte associated protein 4 (CTLA4) blockade.

Along with programmed cell death 1 ligand 1 (PD-L1) and programmed cell death 1 (PD-1), there is another inhibitory mechanism. Cytotoxic antigen, which associated with T lymphocytes (CTLA4), can compete with CD80/86 and inhibit T cell activation [18]. After that, PD1, which are expressed on the surface of T cells, bind to PD-L1 and block immune responses. As a result, T cells do not affect on the tumor and there is no objective response to treatment. Thus, tumor-associated immunosuppressive mechanisms play a key role in blocking the abscopal effect of radiation therapy.

In 2018, James Ellison (USA) and Tasuku Honjo (Japan) received the Nobel Prize for their revolutionary discovery in understanding the mechanism of cancer immunotherapy and the development of tumor immunotherapy. Both scientists in independent studies studied the same phenomenon, and found two different immune checkpoints (control points)-mechanisms for inhibiting $T$ cell activity and suppressing the overall immune response [19]. T cells interact with dendritic cells and form a complex system of many proteins connecting to each other on membranes, or the immune synapse. Some of these protein molecules are co-stimulants and can contribute to enhanced activation of lymphocytes. The discovery of Ellison and Honjo concerned molecules of inhibitors of the $T$ cell response-CTLA-4 and PD-1. Drugs-inhibitors of immune

Table 1. Clinical cases of abscopal effect after RT with immunotherapy according to the search criteria

\begin{tabular}{|c|c|c|c|c|c|c|c|}
\hline Study & Year & Age $(y r) / S e x$ & Primary tumor & RT dose (Gy) & Immunotherapy & Site of abscopal effect & $\begin{array}{l}\text { Time } \\
\text { (mo) }\end{array}$ \\
\hline Wersall et al. [21] & 2006 & $64 / F$ & Kidney & NA & Interferon, interleukin-2 & Lung & 13 \\
\hline Stamell et al. [22] & 2012 & $67 / M$ & Melanoma & $24 \times 3$ & Ipilimumab & LN & NA \\
\hline Postow et al. [23] & 2012 & $33 / F$ & Melanoma & 28.5 & Ipilimumab & Lung & 4 \\
\hline \multirow[t]{2}{*}{ Hiniker et al. [24] } & 2012 & $57 / M$ & Melanoma & $54 \times 3$ & Ipilimumab & Liver, LN & 6 \\
\hline & & $57 / M$ & Melanoma & 54 & Ipilimumab & Liver & 4 \\
\hline Golden et al. [25] & 2013 & $64 / M$ & Lung & 30 & Ipilimumab & Lung, liver, bones & NA \\
\hline Kodama et al. [26] & 2014 & $74 / M$ & Lung & $58 \times 29$ & Bcg-vaccine & Lung & 6 \\
\hline \multirow[t]{11}{*}{ Grimaldi et al. [27] } & 2014 & NA & Melanoma & $30 \times 10$ & Ipilimumab & Liver & 1 \\
\hline & & NA & Melanoma & $30 \times 10$ & Ipilimumab & Pelvic & 1 \\
\hline & & NA & Melanoma & $50 \times 25$ & Ipilimumab & Liver, derma & 4 \\
\hline & & NA & Melanoma & $20 \times 5$ & Ipilimumab & Intestines, derma, lung, LN & 1 \\
\hline & & NA & Melanoma & $30 \times 10$ & Ipilimumab & Liver, ovary & 1 \\
\hline & & NA & Melanoma & $30 \times 10$ & Ipilimumab & Lung, derma, LN & 3 \\
\hline & & NA & Melanoma & $30 \times 10$ & Ipilimumab & Derma, sternum, LN & 1 \\
\hline & & NA & Melanoma & $30 \times 10$ & Ipilimumab & Lung & 1 \\
\hline & & NA & Melanoma & 24 & Ipilimumab & Derma & 1 \\
\hline & & NA & Melanoma & 20 & Ipilimumab & Liver & 2 \\
\hline & & NA & Melanoma & 24 & Ipilimumab & Lung & 1 \\
\hline Michot et al. [28] & 2016 & $33 / \mathrm{M}$ & Lymphoma & 30 & Pembrolizumab & LN & 2 \\
\hline Cong et al. [29] & 2017 & $64 / F$ & Lung & 37.5 & Dendritic cells, cytokine killers & Lung & 10 \\
\hline Shi et al. [30] & 2017 & $67 / F$ & Pancreatic & 45 & $\begin{array}{l}\text { Granulocyte-macrophage colony } \\
\text { stimulating factor }\end{array}$ & Liver & 1 \\
\hline LaPlant et al. [31] & 2017 & $24 / F$ & Kidney & 27 & Ipilimumab & Lung, LN & 7 \\
\hline Sato et al. [32] & 2017 & $54 / \mathrm{M}$ & Stomach & 48 & T-cells, dendritic cells & Abdomen & 2 \\
\hline Britschgi et al. [33] & 2018 & $47 / \mathrm{M}$ & Lung & 18 & Nivolumab & $\mathrm{LN}$ & 3 \\
\hline Tsui et al. [34] & 2018 & $65 / F$ & Melanoma & 24 & Nivolumab & Lung & NA \\
\hline Zhao et al. [35] & 2018 & $65 / M$ & Esophagus & 42 & Pembrolizumab & LN & 2 \\
\hline Rodriguez-Ruiz et al. [36] & 2018 & $68 / M$ & Prostate & 24 & Dendritic cells, hiltonol & Lung & 3 \\
\hline
\end{tabular}

$\mathrm{RT}$, radiation therapy; LN, lymph node; NA, not applicable. 
Table 2. Clinical cases of abscopal effect after RT according to the search criteria

\begin{tabular}{|c|c|c|c|c|c|c|}
\hline Study & Year & Age $(y r) / S e x$ & Primary tumor & RT dose (Gy) & Site of abscopal effect & Time (mo) \\
\hline Ehlers and Fridman [37] & 1973 & $35 / F$ & Thyroid & 40 & Mediastinum & NA \\
\hline Kingsley [38] & 1975 & $28 / \mathrm{M}$ & Melanoma & $14.4 \times 12$ & LN & 2 \\
\hline \multirow[t]{2}{*}{ Antoniades et al. [39] } & 1977 & $40 / \mathrm{M}$ & Lymphoma & $30 \times 20$ & LN & 1 \\
\hline & & $44 / \mathrm{M}$ & Lymphoma & $30 \times 20$ & LN & 1 \\
\hline \multirow[t]{2}{*}{ Fairlamb [40] } & 1981 & $53 / \mathrm{M}$ & Kidney & $21 \times 3$ & Lung & 12 \\
\hline & & $73 / \mathrm{F}$ & Kidney & $40 \times 15$ & Lung & 12 \\
\hline \multirow[t]{2}{*}{ Rees and Ross [41] } & 1983 & $56 / M$ & Lung & $35 \times 10$ & Derma & 1 \\
\hline & & $49 / M$ & Esophagus & $20 \times 20$ & Lung & 6 \\
\hline MacManus [42] & 1994 & $58 / \mathrm{M}$ & Kidney & $20 \times 10$ & Lung, LN & 6 \\
\hline Sham [43] & 1995 & $77 / \mathrm{M}$ & Leukemia & 32.4 & Bones & 1 \\
\hline Ohba et al. [44] & 1998 & $76 / \mathrm{M}$ & Liver & 36 & Bones, hepatocytes & 10 \\
\hline Nam et al. [45] & 2005 & $65 / \mathrm{M}$ & Liver & 30 & Bones, hepatocytes & 10 \\
\hline \multirow[t]{3}{*}{ Golden et al. [25] } & 2006 & $83 / F$ & Kidney & 8 & Lung & 24 \\
\hline & & $69 / \mathrm{M}$ & Kidney & 15 & Lung & 3 \\
\hline & & $55 / F$ & Kidney & 8 & Lung & 5 \\
\hline \multirow[t]{2}{*}{ Takaya et al. [46] } & 2007 & $69 / F$ & Cervix & $50.8 \times 27$ & LN & NA \\
\hline & & & & $24 \times 4$ & & \\
\hline Nakanishi et al. [47] & 2008 & 79/M & Liver & 48 & Liver & NA \\
\hline Lakshmanagowda et al. [48] & 2009 & $65 / F$ & Leukemia & $24 \times 12$ & LN & 1 \\
\hline Cotter et al. [49] & 2011 & $70 / \mathrm{M}$ & Merkel's carcinoma & $12 \times 2$ & Derma & 1 \\
\hline Okuma et al. [50] & 2011 & $63 / \mathrm{M}$ & Liver & $60 \times 27$ & Lung & 1 \\
\hline Tubin et al. [51] & 2012 & $72 / \mathrm{M}$ & Thyroid & $30 \times 3$ & LN & 1 \\
\hline Ishiyama et al. [52] & 2012 & $61 / M$ & Kidney & $18.4 \times 8$ & Lung, bones & 1 \\
\hline Siva et al. [53] & 2013 & $78 / F$ & Lung & 26 & Bones, adrenal glands & 12 \\
\hline Kuroda et al. [54] & 2019 & $76 / F$ & Lung & 60 & Lung & 3 \\
\hline
\end{tabular}

$\mathrm{RT}$, radiation therapy; LN, lymph node; NA, not applicable.

checkpoints have made it possible to achieve certain successes in the immunotherapy of cancer patients. The checkpoint inhibitors CTLA1, PD1, and PDL is rapidly becoming a promising therapeutic area in RT, due to which it is proposed to increase antitumor immunity and increase the frequency of the abscopal effect [18]. The combination of radiation and immune therapy can potentially help overcome tumor-induced immunosuppression, which causes a lack of activating effect of RT on tumor-specific T cells. RT, damaging the DNA of tumor cells, leads to apoptosis, aging and cellular autophagy, induces immunogenic death of tumor cells. This is cross-priming of tumor-specific T cells, the generation of various inflammatory signals that contribute to the activation of dendritic cells [20]. Immunotherapy removes the block from the activation of tumor-specific T cells and leads to a significant antitumor effect and an abscopal effect (Fig. 1).

\section{Clinical Cases}

The abscopal effect was shown in many malignant neoplasms, such as renal cell carcinoma, melanoma, lymphoma, hepatocellular car- cinoma and other types, but this is still a rare and poorly studied phenomenon [12].

As a result of the literature analysis, 35 clinical studies were found in the range from 1973 to 2019 (46 years), which describe 51 cases of the abscopal effect at various locations [21-54] (Tables $1,2)$. It was found that in 33\% (17/51 patients) of cases, the abscopal effect was recorded in melanoma. Also often the primary location of the tumor was the kidney $(18 \%, 9 / 51$ cases) and the lung (14\%, 7/51 cases). The most common localizations of metastases in which the abscopal effect was recorded were the lung (41\%), lymph nodes (31\%), and the liver (15.7\%). It is also worth noting that in only 13 out of 51 cases, multiple regression of tumor metastases was observed.

Nowadays, the literature data are very different regarding depending on the presence or absence of combined treatment (radiation + immunotherapy), so all studies were divided into two groups. The first group included cases with a combination of radiation and immunotherapy (27/51 studies) (Table 1). The second group consisted of patients only with RT (24/51 cases) (Table 2).

In 17 of 27 cases (63\%) in patients of the first group, immuno- 
therapy was carried out with a CTLA4 inhibitor drug, which leads to an increase in the T cell antitumor response, ipilimumab. The average time after which the abscopal effect was recorded is 3.3 months (from 1 to 13 months).

In patients of the second group, the abscopal effect was observed on average after 5.4 months (from 1 to 24 months) (Table 2). In 29\% (7/24 cases) of patients of the second group there was kidney cancer as primary localization, most often the abscopal effect was detected with distant metastases in the lung and lymph nodes (41\% and 32\%, respectively).

After a comparative analysis of the group of patients with the presence of RT alone and the group with combined treatment, the number of cases at the beginning of the 20th century significantly increased with the advent of immunotherapy. Twenty-seven cases were recruited in 13 years, while only with RT 24 cases were recruited in 46 years. Also, the time of manifestation of the abscopal effect with regression of distant metastases was reduced from 5.4 to 3.3 months. The differences here are statistically insignificant ( $p$ $=0.141$, by Student's criterion), but this can be attributed to the small number of samples and the heterogeneity of the drugs used for immunotherapy. Control point inhibitors and CTLA4 give a more frequent abscopal effect (on average after 2.5 months), then other immunotherapy methods.

\section{Discussion and Conclusion}

Despite the long history of the study, the abscopal effect is still a rare and poorly studied phenomenon. However, with the advent of new approaches to immunotherapy, which allows overcoming the tumor associated immunosuppression in combination with RT, the frequency of registration of cases of abscopal effect began to increase significantly. The same conclusion was reached by a number of authors that after the start of using agents on CTLA-4 or the PD-1/PD-L1 axis, the number of registered patients with an abscopal effect increased $[55,56]$. The study of the molecular mechanisms of the RT-induced antitumor immune response is becoming a relevant topic. There is an opportunity to identify predictive markers that allow you to consciously use the combined treatment with the induction of an abscopal effect more consciously. Also identify new targets for drug exposure in order to induce an abscopal effect.

\section{Conflict of Interest}

No potential conflict of interest relevant to this article was reported.

\section{References}

1. McCulloch HD. On the analogy between spontaneous recoveries from cancer and the specific immunity induced by $x$ ray irradiations of the lymphatic glands involved. Br Med J 1908;2:1146-8.

2. Segal G, Leblond CP. Reaction d'alarme produite par l'action des rayons X sur l'abdomen chez le rat. Compt Rend Soc De Biol 1938;129:279.

3. Mole RH. Whole body irradiation; radiobiology or medicine? $\mathrm{Br} J$ Radiol 1953;26:234-41.

4. Andrews JR. Radiobiology of human cancer radiotherapy. Baltimore, MD: University Park Press; 1978.

5. Kelly E, Russell SJ. History of oncolytic viruses: genesis to genetic engineering. Mol Ther 2007;15:651-9.

6. Coley WB. II. Contribution to the knowledge of sarcoma. Ann Surg 1891;14:199-220.

7. Stone HB, Peters $\sqcup$, Milas L. Effect of host immune capability on radiocurability and subsequent transplantability of a murine fibrosarcoma. J Natl Cancer Inst 1979;63:1229-35.

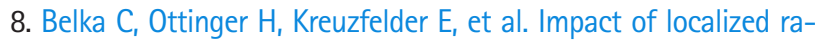
diotherapy on blood immune cells counts and function in humans. Radiother Oncol 1999;50:199-204.

9. Lauber K, Brix N, Ernst A, et al. Targeting the heat shock response in combination with radiotherapy: sensitizing cancer cells to irradiation-induced cell death and heating up their immunogenicity. Cancer Lett 2015;368:209-29.

10. Zelenay S, Reis e Sousa C. Adaptive immunity after cell death. Trends Immunol 2013;34:329-35.

11. Herrera FG, Bourhis J, Coukos G. Radiotherapy combination opportunities leveraging immunity for the next oncology practice. CA Cancer J Clin 2017;67:65-85.

12. Sharma P, Allison JP. Immune checkpoint targeting in cancer therapy: toward combination strategies with curative potential. Cell 2015;161:205-14.

13. Siva S, MacManus MP, Martin RF, Martin OA. Abscopal effects of radiation therapy: a clinical review for the radiobiologist. Cancer Lett 2015;356:82-90.

14. Kroemer G, Galluzzi L, Kepp 0, Zitvogel L. Immunogenic cell death in cancer therapy. Annu Rev Immunol 2013;31:51-72.

15. Apetoh L, Ghiringhelli F, Tesniere A, et al. The interaction between HMGB1 and TLR4 dictates the outcome of anticancer chemotherapy and radiotherapy. Immunol Rev 2007;220:47-59.

16. Tabi Z, Spary LK, Coleman S, Clayton A, Mason MD, Staffurth J. Resistance of CD45RA-T cells to apoptosis and functional impairment, and activation of tumor-antigen specific T cells during radiation therapy of prostate cancer. J Immunol 2010;185:13309. 


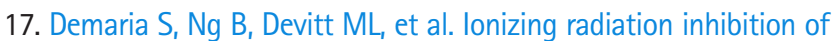
distant untreated tumors (abscopal effect) is immune mediated. Int J Radiat Oncol Biol Phys 2004;58:862-70.

18. Thompson RH, Allison JP, Kwon ED. Anti-cytotoxic T Iymphocyte antigen-4 (CTLA-4) immunotherapy for the treatment of prostate cancer. Urol Oncol 2006;24:442-7.

19. Krummel MF, Allison JP. CD28 and CTLA-4 have opposing effects on the response of T cells to stimulation. J Exp Med 1995;182: 459-65.

20. Eriksson D, Stigbrand T. Radiation-induced cell death mechanisms. Tumour Biol 2010;31:363-72.

21. Wersall PJ, Blomgren H, Pisa P, Lax I, Kalkner KM, Svedman C. Regression of non-irradiated metastases after extracranial stereotactic radiotherapy in metastatic renal cell carcinoma. Acta Oncol 2006;45:493-7.

22. Stamell EF, Wolchok JD, Gnjatic S, Lee NY, Brownell I. The abscopal effect associated with a systemic anti-melanoma immune response. Int J Radiat Oncol Biol Phys 2013;85:293-5.

23. Postow MA, Callahan MK, Barker CA, et al. Immunologic correlates of the abscopal effect in a patient with melanoma. $\mathrm{N}$ Engl J Med 2012;366:925-31.

24. Hiniker SM, Chen DS, Reddy S, et al. A systemic complete response of metastatic melanoma to local radiation and immunotherapy. Transl Oncol 2012;5:404-7.

25. Golden EB, Demaria S, Schiff PB, Chachoua A, Formenti SC. An abscopal response to radiation and ipilimumab in a patient with metastatic non-small cell lung cancer. Cancer Immunol Res 2013;1:365-72.

26. Kodama K, Higashiyama M, Okami J. et al. A possible abscopal effect of post-irradiation immunotherapy in two patients with metastatic lung tumors. Int Canc Conf J 2014;3:122-7.

27. Grimaldi AM, Simeone E, Giannarelli D, et al. Abscopal effects of radiotherapy on advanced melanoma patients who progressed after ipilimumab immunotherapy. Oncoimmunology 2014;3: e28780.

28. Michot JM, Mazeron R, Dercle L, et al. Abscopal effect in a Hodgkin lymphoma patient treated by an anti-programmed death 1 antibody. Eur J Cancer 2016;66:91-4.

29. Cong Y, Shen G, Wu S, Hao R. Abscopal regression following SABR for non-small-cell-lung cancer: a case report. Cancer Biol Ther 2017;18:1-3.

30. Shi F, Wang X, Teng F, Kong L, Yu J. Abscopal effect of metastatic pancreatic cancer after local radiotherapy and granulocyte-macrophage colony-stimulating factor therapy. Cancer Biol Ther 2017;18:137-41.

31. LaPlant Q, Deselm C, Lockney NA, Hsieh J, Yamada Y. Potential abscopal response to dual checkpoint blockade in RCC after reir- radiation using dose-painting SBRT. Pract Radiat Oncol 2017;7: 396-9.

32. Sato H, Suzuki Y, Yoshimoto Y, et al. An abscopal effect in a case of concomitant treatment of locally and peritoneally recurrent gastric cancer using adoptive T-cell immunotherapy and radiotherapy. Clin Case Rep 2017;5:380-4.

33. Britschgi $C$, Riesterer $O$, Burger IA, Guckenberger M, Curioni-Fontecedro A. Report of an abscopal effect induced by stereotactic body radiotherapy and nivolumab in a patient with metastatic non-small cell lung cancer. Radiat Oncol 2018;13:102.

34. Tsui JM, Mihalcioiu C, Cury FL. Abscopal effect in a stage IV melanoma patient who progressed on pembrolizumab. Cureus 2018; 10:e2238.

35. Zhao X, Kang J, Zhao R. Abscopal effect of radiation on lymph node metastasis in esophageal carcinoma: a case report and literature review. Oncol Lett 2018;16:3555-60.

36. Rodriguez-Ruiz ME, Perez-Gracia JL, Rodriguez I, et al. Combined immunotherapy encompassing intratumoral poly-ICLC, dendritic-cell vaccination and radiotherapy in advanced cancer patients. Ann Oncol 2018;29:1312-9.

37. Ehlers G, Fridman M. Abscopal effect of radiation in papillary adenocarcinoma. Br J Radiol 1973;46:220-2.

38. Kingsley DP. An interesting case of possible abscopal effect in malignant melanoma. Br J Radiol 1975;48:863-6.

39. Antoniades J, Brady LW, Lightfoot DA. Lymphangiographic demonstration of the abscopal effect in patients with malignant lymphomas. Int J Radiat Oncol Biol Phys 1977;2:141-7.

40. Fairlamb DJ. Spontaneous regression of metastases of renal cancer: a report of two cases including the first recorded regression following irradiation of a dominant metastasis and review of the world literature. Cancer 1981;47:2102-6.

41. Rees GJ, Ross CM. Abscopal regression following radiotherapy for adenocarcinoma. Br J Radiol 1983;56:63-6.

42. MacManus MP, Harte RJ, Stranex S. Spontaneous regression of metastatic renal cell carcinoma following palliative irradiation of the primary tumour. Ir J Med Sci 1994;163:461-3.

43. Sham RL. The abscopal effect and chronic lymphocytic leukemia. Am J Med 1995;98:307-8.

44. Ohba K, Omagari K, Nakamura T, et al. Abscopal regression of hepatocellular carcinoma after radiotherapy for bone metastasis. Gut 1998:43:575-7.

45. Nam SW, Han JY, Kim Jl, et al. Spontaneous regression of a large hepatocellular carcinoma with skull metastasis. J Gastroenterol Hepatol 2005;20:488-92.

46. Takaya M, Niibe $Y$, Tsunoda $S$, et al. Abscopal effect of radiation on toruliform para-aortic lymph node metastases of advanced uterine cervical carcinoma: a case report. Anticancer Res 2007; 
27(1B):499-503.

47. Nakanishi M, Chuma M, Hige S, Asaka M. Abscopal effect on hepatocellular carcinoma. Am J Gastroenterol 2008;103:1320-1.

48. Lakshmanagowda PB, Viswanath L, Thimmaiah N, Dasappa $L$, Supe SS, Kallur P. Abscopal effect in a patient with chronic lymphocytic leukemia during radiation therapy: a case report. Cases J 2009;2:204.

49. Cotter SE, Dunn GP, Collins KM, et al. Abscopal effect in a patient with metastatic Merkel cell carcinoma following radiation therapy: potential role of induced antitumor immunity. Arch Dermatol 2011;147:870-2.

50. Okuma K, Yamashita H, Niibe Y, Hayakawa K, Nakagawa K. Abscopal effect of radiation on lung metastases of hepatocellular carcinoma: a case report. J Med Case Rep 2011;5:111.

51. Tubin S, Casamassima F, Menichelli C, Pastore G, Fanelli A, Crisci R. A case report on metastatic thyroid carcinoma: radiation-induced bystander or abscopal effect. J Cancer Sci Ther 2012;4: 408-11.

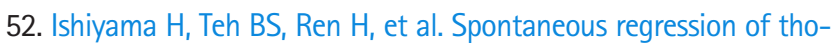
racic metastases while progression of brain metastases after stereotactic radiosurgery and stereotactic body radiotherapy for metastatic renal cell carcinoma: abscopal effect prevented by the blood-brain barrier? Clin Genitourin Cancer 2012;10:196-8.

53. Siva S, Callahan J, MacManus MP, Martin O, Hicks RJ, Ball DL. Abscopal [corrected] effects after conventional and stereotactic lung irradiation of non-small-cell lung cancer. J Thorac Oncol 2013;8:e71.

54. Kuroda A, Tabuchi $T$, Iwami $E_{1}$ et al. Abscopal effect of radiation on multiple lung metastases of lung adenocarcinoma: a case report. BMC Cancer 2019;19:336.

55. Brahmer JR, Tykodi SS, Chow LQ, et al. Safety and activity of anti-PD-L1 antibody in patients with advanced cancer. N Engl J Med 2012;366:2455-65.

56. Reynders K, Illidge T, Siva S, Chang JY, De Ruysscher D. The abscopal effect of local radiotherapy: using immunotherapy to make a rare event clinically relevant. Cancer Treat Rev 2015;41:503-10. 